\title{
Single Lamella Nanoparticles of Polyethylene
}

\author{
C. H. M. Weber, ${ }^{\dagger}$ A. Chiche, and G. Krausch*, ${ }^{\star}$ \\ Physikalische Chemie II, University of Bayreuth, Universitätsstrasse 30, \\ 95440 Bayreuth, Germany
}

\section{S. Rosenfeldt and M. Ballauff*}

Physikalische Chemie I, University of Bayreuth, Universitätsstrasse 30, 95440 Bayreuth, Germany

\section{Harnau}

Max-Planck-Institut für Metallforschung, Heisenbergstrasse 3, 70569 Stuttgart, Germany, and Institut für Theoretische und Angewandte Physik, Universität Stuttgart, Pfaffenwaldring 57, 70569 Stuttgart, Germany

\author{
I. Göttker-Schnetmann, Q. Tong, and S. Mecking* \\ Department of Chemistry, University of Konstanz, Universitätssstrasse 10, \\ 78457 Konstanz, Germany
}

\begin{abstract}
We present a complete analysis of the structure of polyethylene (PE) nanoparticles synthesized and stabilized in water under very mild conditions $\left(15^{\circ} \mathrm{C}, 40 \mathrm{~atm}\right)$ by a nickel-catalyzed polymerization in aqueous solution. Combining cryogenic transmission electron microscopy (cryo-TEM) with X-ray scattering, we demonstrate that this new synthetic route leads to a stable dispersion of individual PE nanoparticles with a narrow size distribution. Most of the semicrystalline particles have a hexagonal shape (lateral size $25 \mathrm{~nm}$, thickness $9 \mathrm{~nm}$ ) and exhibit the habit of a truncated lozenge. The combination of cryo-TEM and small-angle X-ray scattering demonstrates that the particles consist of a single crystalline lamella sandwiched between two thin amorphous polymer layers ("nanohamburgers"). Hence, these nanocrystals that comprise only ca. 14 chains present the smallest single crystals of PE ever reported. The very small thickness of the crystalline lamella (6.3 nm) is related to the extreme undercooling (more than $100^{\circ} \mathrm{C}$ ) that is due to the low temperature at which the polymerization takes place. This strong undercooling cannot be achieved by any other method so far. Dispersions of polyethylene nanocrystals may have a high potential for a further understanding of polymer crystallization as well as for materials science as, e.g., for the fabrication of extremely thin crystalline layers.
\end{abstract}

Polyethylene (PE) is a commodity polymer that has become ubiquitous over the past several decades because of its low price and good mechanical properties. ${ }^{1}$ Hence, the number of applications of the material is huge and many millions of tons are produced worldwide annually. However, PE has hardly played any role in the field of nanotechnology. This is due to the problem that PE is produced either by free radical polymerization under high pressure and temperature or with metal-organic catalysts working exclusively under strictly water-free conditions. Polymer nanoparticles and their

\footnotetext{
*Corresponding authors: krausch@uni-mainz.de; Matthias.Ballauff@ uni-Bayreuth.de; Stefan.Mecking@uni-Konstanz.de.

$\dagger$ Present address: Department of Chemistry, University of Sheffield, Sheffield, S3 7HF, U.K.

$\doteqdot$ Present address: Universitaet Mainz, 55128 Mainz, Germany.
}

composites with inorganic compounds, however, are very often produced in aqueous systems. ${ }^{2}$

Recently, it was demonstrated that ethylene can be polymerized in aqueous systems in a catalytic fashion by $\mathrm{Ni}(\mathrm{II})$ complexes. ${ }^{3-6}$ By virtue of this novel synthesis, long chains of polyethylene can be generated in a well-controlled environment and at ambient temperature. Thus, it could be shown that aqueous PE dispersions can be produced. This novel way of polymerization hence opens the way for the creation of nanostructures made from PE. Up to now, the particles synthesized in this way were semicrystalline and for the largest part consisted of stacks of several crystalline lamellae. ${ }^{6}$

Here we demonstrate for the first time that well-defined polyethylene nanoparticles consisting of a single lamella can 
be generated in this way selectively. The purpose of the present work is 2-fold: First, we demonstrate that the catalytic polymerization of ethylene in an aqueous medium with judicious choice of the parameters can lead to welldefined colloidal particles. Second, the analysis of the particles thus obtained may be highly useful for fundamental studies of chain folding in polymer crystallization. ${ }^{7,8} \mathrm{Up}$ to now, most work related to the crystallization of polyethylene has been done by cooling down from the molten or dissolved state. ${ }^{8}$ This imposes narrow limits to the conditions under which crystallization can take place. We are using here a new way to prepare semicrystalline PE under extremely mild conditions of pressure and temperature. During this process, nanoparticles exclusively made of polyethylene are formed by the chains as soon as they polymerize (aqueous monomer solution). In principle, the crystallization could start while the chains are still growing at $15{ }^{\circ} \mathrm{C}$. However, the fast polymerization process and the slow nucleation in confined nanoparticles ${ }^{9}$ will most probably prevent the crystallization from starting before the whole particle is formed. It is thus a melt crystallization that takes place in each nanoparticle with an extraordinary degree of supercooling which could not be achieved by any method known so far. We therefore compare our present results to the by now classical results on chain crystallization of $\mathrm{PE}$ from the melt or from solutions.

The analysis presented here employs a combination of cryogenic transmission electron microscopy (cryo-TEM ${ }^{10,11}$ ) and small-angle X-ray scattering (SAXS ${ }^{12,13}$ ). While cryoTEM allows us to study the shape of the particles in a shockfrozen solution, the analysis by SAXS leads to the in situ determination of the internal structure of the particles. By this unique combination of methods, we show unambiguously that the PE particles produced here are single faceted nanocrystals and consist of a lamella covered by an amorphous phase in which the PE chains fold back.

Experimental Section. The system was prepared by catalytic polymerization as delineated previously. ${ }^{14}$ The original dispersion as obtained only contains PE (3.6 wt \%) and just enough of the surfactant sodium dodecyl sulfate (SDS) to stabilize the particles against coagulation. Unlike typical free radical emulsion polymerization, ${ }^{14,15}$ the particles only appear as a result of the polymerization in a nonsolvent for the polymer. The surface tension of $65 \mathrm{mN} / \mathrm{m}$ of the dispersion demonstrates that virtually all the surfactant is adsorbed onto the particles; no free micelles are present in the system. The molecular weight of the resulting polymer is $2 \times 10^{5} \mathrm{~g} / \mathrm{mol}$ and the polydispersity as given by the ratio of the weight-average to the number average molecular weight of the polymer is 2 . This finding points to a welldefined process of polymerization.

Specimens for cryo-TEM were prepared by vitrification of a thin liquid film of the diluted dispersion supported by a copper grid in liquid ethane. Examinations were carried out at temperatures around $90 \mathrm{~K}$. Hence, the particles are analyzed in situ, that is, in the suspension. Moreover, no staining agent has been used to enhance the contrast between

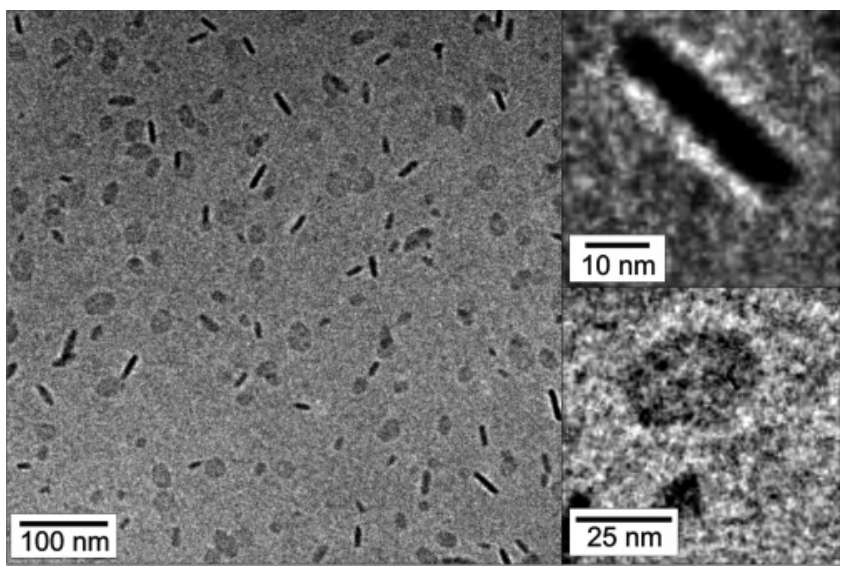

Figure 1. Cryo-TEM micrograph of the polyethylene particles in aqueous dispersion. The weight concentration of the particles was $0.4 \mathrm{wt} \%$. The gray background is the low-density amorphous ice ${ }^{16}$ in which the particles are dispersed. The particles are flat platelets, appearing as rods when parallel to the electron beam (upper inset) and often as hexagons when more orthogonal to the electron beam (lower inset).

the objects and the surrounding medium (for details of the general procedure cf. ref 11).

Results and Discussion. Figure 1 displays a micrograph of the particles in dilute aqueous solution as obtained by cryo-TEM. The dispersion consists of flat platelets with a rather narrow size distribution. The different gray scales for different particles can be easily rationalized by different viewing angles: If the platelets are nearly parallel to the electron beam, the length of the optical path through the particles is much longer than for nearly perpendicular arrangement. From the cryo-TEM images we determined a lateral dimension (pseudodiameter) of $25.4( \pm 4.3) \mathrm{nm}$ and a corresponding thickness of the platelets of $6.3( \pm 0.8) \mathrm{nm}$. These figures have been derived from the image analysis of 67 particles with approximately perpendicular orientation to the electron beam.

We interpret the observed nanoplatelets as single lamellae of polyethylene with a thickness $L_{\mathrm{c}}$ of $6.3 \mathrm{~nm}$. The crystallinity of the nanoparticles is confirmed by wide-angle X-ray scattering (WAXS) measurements performed on the dispersion as obtained at room temperature. The WAXS diffraction pattern exhibits the two Bragg peaks ((110) and (200)) of polyethylene and indicates that the particles are monocrystalline (the Scherrer length being virtually the same as the particle pseudo-diameter as measured from cryo-TEM). ${ }^{17}$

It is difficult to characterize the exact shape of these nanocrystals due to the unknown tilt of each particle relative to the electron beam and a certain disparity of shapes as shown by Figure 1. However, most particles have rather straight edges (faceted lamellae), and many of these appear hexagonal as shown in the figure inset. We can recognize in this shape the truncated lozenge habit of PE single crystals, defined by the crystalline planes $\{110\}$ and $\{100\}$ and typically obtained by crystallization from the melt ${ }^{18,19}$ or from solutions at relatively high temperature (more than ca. $\left.70{ }^{\circ} \mathrm{C}\right) .{ }^{20,21}$ No particle with a lozenge shape has been found in any of the micrographs. The possible origin of the morphology will be discussed later. 
The crystalline lamellae visible in Figure 1 are expected to be covered by an amorphous layer as polyethylene is a semicrystalline polymer. ${ }^{8}$ However, it is not possible to detect this by cryo-TEM. This is due to the fact that the electron density of amorphous PE is virtually the same as that of the low density amorphous ice (see below). Hence, there is not a sufficient contrast between a possible amorphous layer and the surrounding medium. However, we shall treat this problem as outlined in the following by applying contrast variation SAXS as an analytical tool. Figure 1 also demonstrates that the particles are well-dispersed in the aqueous medium; virtually no aggregates are found. This is a prerequisite for a meaningful investigation by scattering methods in solution.

In the following we describe the analysis of the particles by SAXS. The intensity $I(q)$ as the function of the magnitude $q$ of the scattering vector $(q=(4 \pi / \lambda) \sin (\theta / 2) ; \lambda$, wavelength of radiation; $\theta$, scattering angle) is sensitive toward the difference between the electron density of the particle and the electron density $\rho_{\mathrm{m}}$ of the surrounding medium. Contrast variation in SAXS is achieved by varying the electron density of the medium by adding an adequate contrast agent. SAXS data are then recorded for different values of the adjusted contrast. ${ }^{13,22}$ The scattering intensity $I(q)$ of $N$ particles per volume $V$ can be rendered as follows ${ }^{12,13,22}$

$$
I(q)=\frac{N}{V} I_{0}(q) S(q)=\phi(\Delta \rho)^{2} V_{\mathrm{p}} P(q) S(q)
$$

where $V_{\mathrm{p}}$ denotes the volume of the solute particle. Equation 1 hence suggests to normalize the intensity to $\phi$, the volume fraction of the dispersed particles. The quantity $\Delta \rho$ is the contrast of the dissolved particles given by the difference of averaged scattering length density of the particle $\bar{\rho}$ and of the scattering length density of the solvent $\rho_{\mathrm{m}} . P(q)$ is the form factor describing the shape and the structure of a single particle. The structure factor $S(q)$ includes the scattering contributions due to interparticle interactions (see below). The scattering intensity of a single particle is given by $I_{0}(q)$.

The SAXS intensities of the polyethylene nanoparticles were measured at six different contrasts starting from a stock solution of $\phi=0.017$ of the nanoparticles dispersed in pure water (see Figure 2). The different contrasts are adjusted by adding different amounts of sucrose. ${ }^{13}$ The volume fraction of added sucrose $\phi_{\mathrm{S}}$ is increased from $\phi_{\mathrm{S}}=0.0$ (lowest contrast) to $\phi_{\mathrm{S}}=0.378$ (highest contrast) while the corresponding volume fraction of the nanoparticles decreased from $\phi=0.017$ to $\phi=0.007$.

Figure 2a demonstrates that varying the contrast leads to marked differences in the scattering intensities. In particular, the maxima of the scattering intensities are shifted in a characteristic manner when changing the contrast $\Delta \rho$. All scattering intensities are slightly depressed at lowest $q$ values. This points immediately to the influence of mutual interaction as expressed through $S(q)$. Previous work has shown, however, that $S(q)$ approaches unity for higher scattering angles (see the discussion of this point in ref 13). Hence,
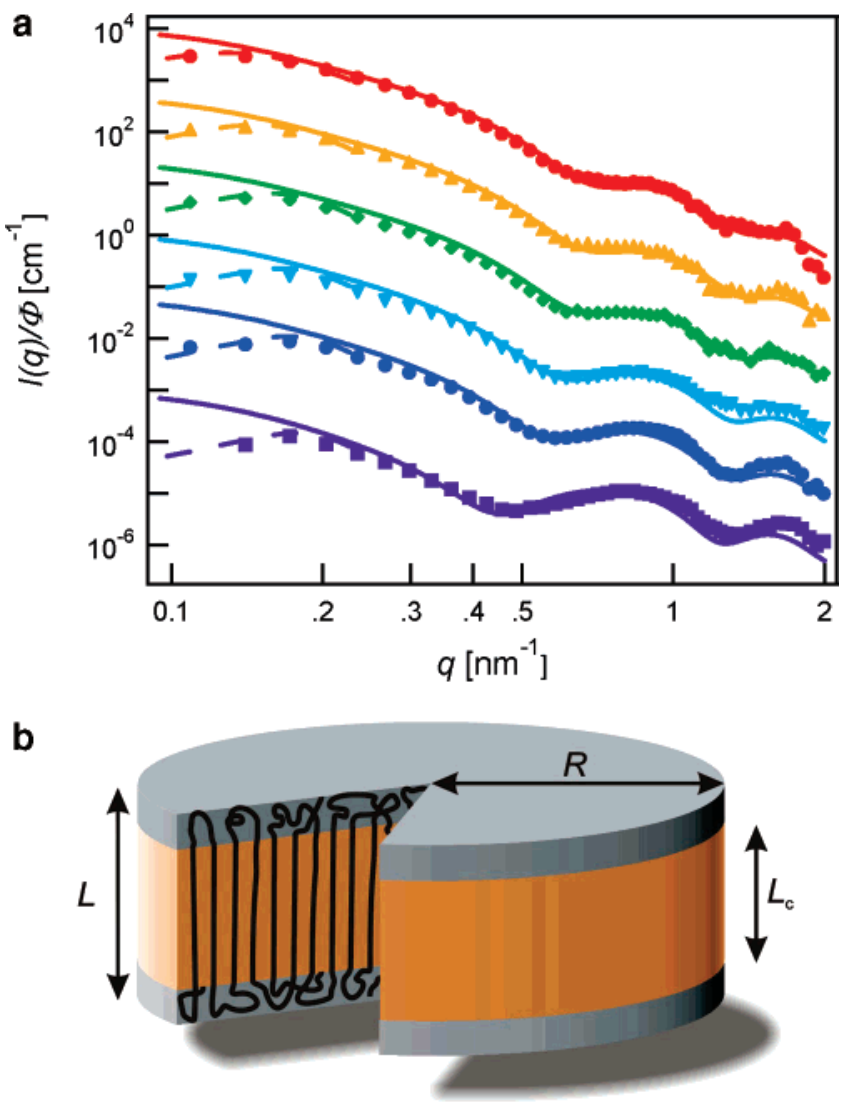

Figure 2. Analysis of the platelets by SAXS. (a) Measured scattering intensity $I(q) / \phi$ of polyethylene nanoparticles as a function of the magnitude of the scattering vector $q$ (symbols). All intensities have been normalized to the volume fraction $\phi$ of the particles in the dispersion. The volume fraction of the added sucrose increases from bottom to top $(0,6.2,10.3,18.0,25.4,37.8$ vol \%) while the volume fraction $\phi$ of the nanoparticles decreases from bottom to top $(1.73,1.56,1.44,1.23,1.02,0.68 \mathrm{vol} \%)$. The five lowermost intensities are shifted down by a factor of $10,10^{2}, 10^{3}, 10^{4}, 10^{5}$, respectively. The solid lines represent the result of the modeling of the SAXS data assuming a dispersion of noninteracting polydisperse disks according to eqs 1 and $2(S(q)=1)$. The short dashed lines $\left(q<0.25 \mathrm{~nm}^{-1}\right)$ represent the scattering intensity calculated for a dispersion of interacting disks as obtained from the PRISM integral equation theory. The differences between the dotted and solid lines reflect the intermolecular interaction between the nanoparticles. (b) Modeling of the SAXS data in terms of a circular disk ("nanohamburger").

for $q>0.2 \mathrm{~nm}^{-1}$ the influence of mutual interaction can be disregarded and the resulting intensity is solely related to the spatial structure of the dispersed particles. This means that the measured intensity is given by $I_{0}(q)$ (cf. eq 1). The correction for the low-angle region can be done by calculating $S(q)$ from the shape of the articles. This point will be discussed further below.

The cryo-TEM micrograph displayed in Figure 1 shows that the particles are platelets with a pseudoradius of 12$13 \mathrm{~nm}$ and a thickness of $6.3 \mathrm{~nm}$. As argued above, however, the cryo-TEM micrographs show only the crystalline lamella. Since polyethylene is a semicrystalline polymer, an amorphous layer in which the chains fold back must exist on both sides of the lamella. Hence, there must be a thin amorphous layer on both sides of the particles that needs to be taken into account. 
The analysis of the SAXS intensities for $q>0.2 \mathrm{~nm}^{-1}$ can therefore be performed as follows: The average scattering length density $\bar{\rho}$ of the solute was calculated to $\bar{\rho}=$ 330 electrons per $\mathrm{nm}^{3}$ from the experimentally determined specific volume $\left(v_{2}=1.042 \mathrm{~cm}^{3} / \mathrm{g}\right)$ and the chemical composition of polyethylene. The quantity $\bar{\rho}$ obtained was used to calculate the contrast $\Delta \rho$ for each intensity shown in Figure 2a. Except for the region of smallest angles, the measured SAXS intensity is only sensitive toward the density distribution along the layer normal. ${ }^{12}$ Hence, the SAXS intensities derived from the measurements carried out at different contrasts were modeled in terms of a circular platelet shown in Figure $2 \mathrm{~b}$. The scattering intensity of such a "hamburger" is given by

$$
\begin{gathered}
I_{0}(q)=\int_{0}^{1} \mathrm{~d} \alpha F^{2}(q, \alpha) \\
F(q, \alpha)=2 \pi R^{2} \frac{J_{1}\left(q R \sqrt{1-\alpha^{2}}\right)}{q R \sqrt{1-\alpha^{2}}}\left(L \frac{\sin (q \alpha L / 2)}{q \alpha L / 2} \Delta \rho_{1}+\right. \\
\left.L_{\mathrm{c}} \frac{\sin \left(q \alpha L_{\mathrm{c}} / 2\right)}{q \alpha L_{\mathrm{c}} / 2}\left(\Delta \rho_{\mathrm{c}}-\Delta \rho_{1}\right)\right)
\end{gathered}
$$

Here $R$ denotes the radius of the disks, $L$ is the overall thickness of the particles, and $L_{\mathrm{c}}$ denotes the thickness of the crystalline lamella. The contrasts $\Delta \rho_{1}$ and $\Delta \rho_{\mathrm{c}}$ are the contrast of the outer two disks and of the inner disk, respectively, and $J_{1}(x)$ denotes the Bessel function of first order. The polydispersity of the particles estimated from the cryo-TEM micrographs was taken into account in the fitting using a Gaussian distribution ${ }^{23}$ in radius and thickness.

The solid lines in Figure 2a display the optimal fits thus obtained. For the sake of clarity the scattering intensities related to different contrast have been shifted vertically. The overall dimensions following from this fit are a weightaverage radius $R_{\mathrm{w}}=12 \mathrm{~nm}$ and a weight-average thickness $L_{\mathrm{w}}=9 \mathrm{~nm}$. The standard deviations of the radius was $6 \mathrm{~nm}$ and for the thickness $1 \mathrm{~nm}$. The thickness of the crystalline layer is $L_{\mathrm{c}}=6.3 \mathrm{~nm}$. From these parameters the volume fraction of the crystalline part can be determined to $\phi_{\text {cryst }}=$ 0.70 . From the particle volume, the polyethylene density, ${ }^{24}$ and the chain molecular weight, we estimate each particle is made up of ca. 14 chains. Moreover, from the fit of the entire series of SAXS intensities the contrast results to $\Delta \rho_{1}=309$ electrons $/ \mathrm{nm}^{3}$ for the amorphous part and $\Delta \rho_{\mathrm{c}}=$ 333 electrons $/ \mathrm{nm}^{3}$ for the crystalline part.

As mentioned above, the electron densities of the crystalline and amorphous parts of the particles can now be used to understand the cryo-TEM micrograph in detail. The value for $\Delta \rho_{1}$ is close to the electron density of low density amorphous ice ${ }^{16}$ (314 electrons $/ \mathrm{nm}^{3}$, cryo-TEM background; cf. the discussion of Figure 1). Therefore we can conclude that the amorphous parts are virtually matched in the cryoTEM.

The interaction between different particles that is embodied in the structure factor $S(q)$ (see eq 1) remains to be discussed. The structure factor that leads to a slight depression of the

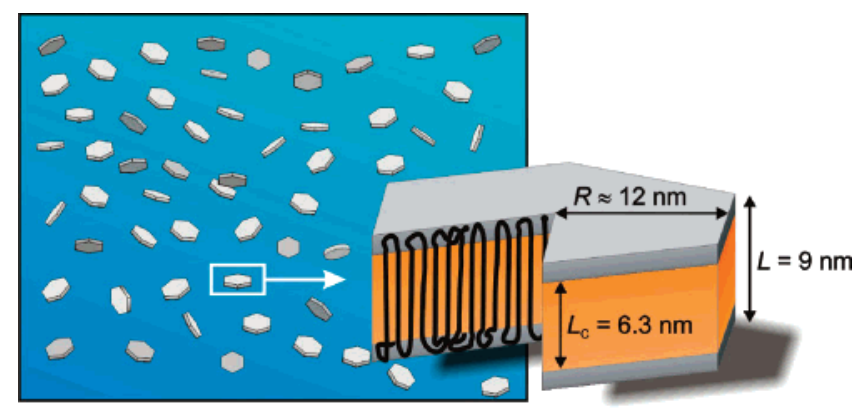

Figure 3. Sketch of the polyethylene nanoparticles in water. These particles consist of a single lamella with a thickness $L_{\mathrm{c}}$ of $6.3 \mathrm{~nm}$ sandwiched between two amorphous sheets resulting in an overall thickness of $9 \mathrm{~nm}$ (including the amorphous layers). As suggested by Figure 1, many of these nanocrystals exhibit a truncated lozenge habit with a lateral dimension $R$. Colloidal stability of the particles is brought about by adsorbed molecules of the charged surfactant sodium dodecyl sulfate (SDS). For the sake of clarity, the adsorbed SDS-molecules are not shown.

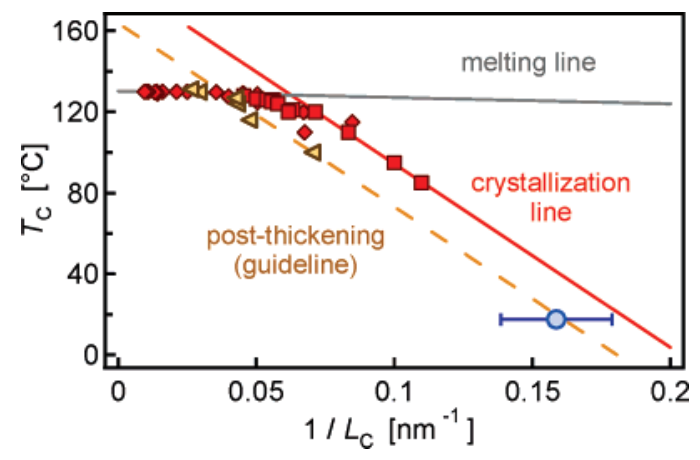

Figure 4. Crystallization temperature $T_{\mathrm{C}}$ as a function of the inverse of the lamellar thickness $L_{\mathrm{c}}$ for isothermally crystallized PE bulk samples from the literature. Depending on $T_{\mathrm{C}}$, the data fall on either the melting line or the crystallization line $\left(T_{\mathrm{C}}{ }^{\infty}\right)$ defined by the Gibbs-Thomson equation (eq 3). ${ }^{7}$ After crystallizing, linear PE lamellae are known to reorganize toward the equilibrium state (stretched chains) and become thicker (shift of the crystallization line). The nanoparticles (open blue circle) clearly went through lamellar thickening after crystallization at room temperature. The full symbols correspond to the initial lamellar thickness (squares from ref 28; diamonds from ref 8). The open triangles correspond to samples with already thickened lamellae due to a longer time at $T_{\mathrm{C}}$ (from ref 29).

measured intensity at lowest scattering angles (see Figure 2a) can be calculated directly from the shape of a single colloidal particle and the interaction potential using the "polymer reference interaction site model" (PRISM) integral equation theory (see refs $25-27$ and references therein). As input parameters the overall dimensions of the disks $(R=$ $12 \mathrm{~nm}, L=9 \mathrm{~nm}$ ), the experimental volume fraction $\phi$, the polydispersity of the particles, and a screened Coulomb interaction with an effective charge $Z=-25$ e of an individual polyethylene nanoparticle have been used.

In Figure 2a experimental scattering intensities are compared with the theoretical results. For small magnitudes of the scattering vectors $q$ the calculated scattering intensities for noninteracting particles, i.e., $S(q)=1$ (solid lines), on the one hand, and the integral equation results for interacting particles (short dashed lines) as well as the experimental data (symbols) on the other hand deviate due to strong repulsive 

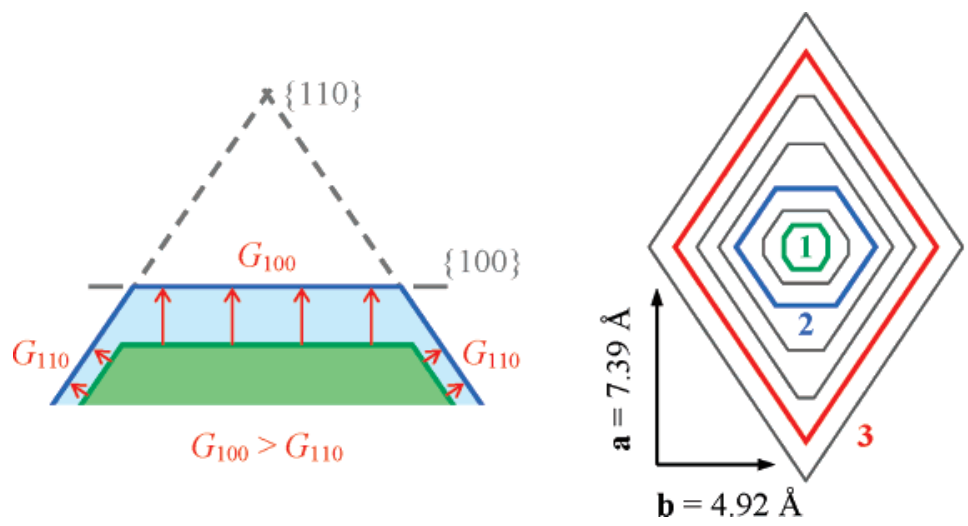

Nascent crystal (1)

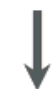

Nano-crystal (2)

truncated lozenge

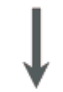

Macro-crystal (3)

lozenge

Figure 5. Schematic growth of a nascent nanocrystal and the potential result on its shape. The faces $\{100\}$ should slowly disappear as they grow faster than the $\{110\}$, as shown on the left side, resulting in a macrocrystal with a lozenge shape defined by $\{110\}$. However, as the crystals stay very small (the distance between their center and the growth front containing less than 25 crystallographic units), the faces $\{100\}$ remain in these nanoparticles, while the faces $\{010\}$ have already vanished (faster grow rate $G$ ). This would explain the truncated lozenge habit of the nanocrystals.

interactions between the particles. The observed decrease of the scattering intensity at small scattering vectors is considerably more pronounced than that for fluids consisting of hard colloidal disks (see Figure 3 in ref 27). From this result we can conclude that the colloidal stability is achieved by electrostatic repulsion between the particles brought about by the adsorbed surfactant SDS. For the sake of clarity, however, the adsorbed SDS molecules are not shown in Figures $2 b$ and 3 . For the system under consideration we have estimated that each polyethylene nanoparticle is surrounded by a layer consisting of a few hundred surfactant molecules.

In summary, a full structural analysis can be achieved by the present combination of cryo-TEM and SAXS. The above discussion demonstrates that these techniques supplement each other in a nearly ideal fashion to elucidate the structure of these nanocrystals stabilized in water, as depicted in Figure 3.

Compared with literature studies of bulk PE, the lamellar thickness $L_{\mathrm{c}}$ is very small which is due to the low temperature of synthesis $\left(15^{\circ} \mathrm{C}\right)$. The crystallization has thus occurred more than $100{ }^{\circ} \mathrm{C}$ below the melting point of polyethylene. This extreme supercooling is not accessible by any other method used so far for the crystallization of polyethylene. It results in a higher degree of chain folding into thinner lamellae, as shown in Figure 4. Here the crystallization temperature $T_{\mathrm{C}}$ is plotted versus the lamellar thickness $L_{\mathrm{c}}$ as suggested by the Gibbs-Thomson equation (see the discussion in ref 7)

$$
T_{\mathrm{c}}=T_{\mathrm{c}}^{\infty}\left(1-\frac{2 \sigma}{\Delta h L_{\mathrm{c}}}\right)
$$

where $\Delta h$ is the heat of fusion, $\sigma$ the surface free energy of the lamella, and $T^{\infty}$ the temperature limit resulting in fully stretched chains. The lamellar thickness we obtained is slightly thicker than the initial fold length expected for room temperature (crystallization line), due to postcrystallization reorganization (lamellar thickening). A similar thickening has been found by other authors in bulk samples. ${ }^{29}$ The thicken- ing of such a small isolated lamellae should however be limited by their edge energy ${ }^{30}$ even after annealing at higher temperature.

As discussed above, most of these nanocrystals are faceted. The shape of a macroscopic polymer crystal results from an anisotropic growth rate and usually corresponds to the slowest crystallographic planes while the fastest growing planes will disappear during the crystal growth. ${ }^{18}$ Faceting is then surprising for such extremely small crystals, the distance between the original nucleus (center), and the growth front containing less than 25 crystalline orthorhombic cells.

The basic habit of a PE crystal is a lozenge, defined by the slowest $\{110\}$ planes. However, lozenges have only been obtained at low temperatures so far only accessible by solution crystallization. ${ }^{21}$ At higher temperatures, in melt ${ }^{19,31}$ or solution crystallization, ${ }^{20,21}$ the $\{100\}$ planes are slowed down by roughening or pinning (multiple secondary nucleation) and the $\{110\}$ lozenge is truncated by $\{100\}$ facets. ${ }^{31,32}$ Despite the extreme undercooling realized here, many nanocrystals have a truncated lozenge habit while no full lozenge shape has been found.

At first, this could clearly indicate that pinning or roughening occurs during the crystallization, resulting in a lower growth rate of the $\{100\}$ faces. This would be clear evidence that no lozenge habit can be obtained from PE melt crystallization even at high undercooling. However, the truncated lozenge habit may also result from the very small size of these nanocrystals and thus their limited growth. The surface/volume ratio is indeed very high for such a small crystal and could result in an unusual equilibrium shape. A more simple explanation is that the $\{100\}$ faces did not yet disappear despite a faster growth than the $\{110\}$ faces because of the very short growing time of this almost nascent crystal. The $\{010\}$ planes with a much higher growth rate have already vanished. This scenario is depicted in Figure 5.

If the truncated lozenge habit results from a slowing down of the $\{100\}$ faces by pinning or roughening even at this extreme undercooling, it should "survive" to a subsequent growth, with even a possible curvature of $\{100\}$ faces. ${ }^{31,32}$ It would not be the case if the extreme size of these 
nanocrystals is involved through the high surface/volume ratio or the very short growth history. An investigation of slightly larger particles should solve this question.

In our knowledge, these are the smallest PE single crystals ever reported, made of ca. 14 chains crystallized. Together with the extreme undercooling of this melt crystallization, this unique system should shed some light in the polymer crystallization process, as shown by the above discussion.

Conclusion. In conclusion, we have shown that welldefined and perfectly dispersed nanoparticles of the cheapest polyolefin, namely polyethylene, can be made in water. The combination of cryo-TEM and SAXS demonstrates that these particles are single nanocrystals consisting of a single crystalline lamella covered by thin amorphous layers. The small thickness $L_{\mathrm{c}}$ of the lamellae $(6.3 \mathrm{~nm})$ matches with extrapolated literature data and results from the enormous supercooling during crystallization. The truncated lozenge shape exhibited by most of these crystals is explained as a consequence of the extremely limited size of these nascent crystals (pseudoradius $12 \mathrm{~nm}$ ). Hence, due to their unusual size, structure, and thermal history, the particles analyzed herein will contribute to fundamental and long-standing issues of polymer crystallization. Moreover, this novel type of dispersion opens the way to interesting applications as, e.g., for environmentally benign coatings and thin films based on polyethylene.

Acknowledgment. We thank A. Lovinger for highly useful comments. Financial support from the Deutsche Forschungsgemeinschaft, SFB 481, Bayreuth, the International Research Training Group "Soft Condensed Matter", Konstanz/Strasbourg/Grenoble, and the European Community's "Marie-Curie Actions" under Contract MRTN-CT2004-504052 [POLYFILM] is gratefully acknowledged. S.M. is indebted to the Fonds der Chemischen Industrie for financial support.

Note Added after ASAP Publication. There were spelling errors in the first sentence of the version of the paper published June 12, 2007; the corrected version was published ASAP June 18, 2007.

\section{References}

(1) Elias, H.-G. An introduction to polymer science; VCH: Weinheim, 1997.

(2) Odian, G. Principles of polymerization, 3rd ed.; J. Wiley: New York, 1991.

(3) Bauers, F. M.; Mecking, S. Angew. Chem. 2001, 113, 3112.

(4) Bauers, F. M.; Mecking, S. Angew. Chem., Int. Ed. 2001, 40, 3020.

(5) Soula, R.; et al. Macromolecules 2001, 34, 2022.

(6) Bauers, F. M.; Thomann, R.; Mecking, S. J. Am. Chem. Soc. 2003, 125,8838 .

(7) Strobl, G. Prog. Polym. Sci. 2006, 31, 398.

(8) Wunderlich, B. Macromolecular Physics; Academic Press: New York, 1973; Vol. 1.

(9) Loo, Y.-L.; Register, R. A.; Ryan, A. J. Phys. Rev. Lett. 2000, 84, 4120 .

(10) Li, Z.; Kesselmann, E.; Talmon, Y.; Hillmyer, M. A.; Lodge, T. P. Science 2004, 306, 98.

(11) Wittemann, A.; Drechsler, M.; Talmon, Y.; Ballauff, M. J. Am. Chem. Soc. 2005, 127, 9688.

(12) Guinier, A.; Fournet, G. Small angle scattering of X-rays; John Wiley: New York, 1955.

(13) Dingenouts, N.; Ballauff, M. Acta Polym. 1993, 44, 178.

(14) Göttker-Schnetmann, I.; Korthals, B.; Mecking, S. J. Am. Chem. Soc. 2006, 128,7708

(15) Taden, A.; Landfester, K. Macromolecules 2003, 36, 4037.

(16) Petrenko, V. F.; Whitworth, R. W. Physics of Ice; Oxford University Press: Oxford, 1999.

(17) WAXS measurements were performed at the beamline BM 26 at the ESRF, Grenoble. We thank Martin Rosenthal for performing these measurements.

(18) Keller, A. Rep. Prog. Phys. 1968, 31, 623.

(19) Martinez-Salazar, J.; Barham, P. J.; Keller, A. Polym. Sci. Polym. Phys. Ed. 1984, 22, 1085.

(20) Bassett, D. C.; Keller, A. Philos. Mag. J. Sci. 1962, 7, 1553.

(21) Organ, S. J.; Keller, A. Mater. Sci. 1985, 20, 1571.

(22) Rosenfeldt, S.; et al. Macromolecules 2002, 35, 8098.

(23) As discussed in detail in ref 13 the exact type of distribution is of minor importance for the fit if its breadth is not too large.

(24) The density of polyethylene as taken from the literature: $0.98 \mathrm{~g} / \mathrm{cm}^{3}$ (crystalline) and $0.91 \mathrm{~g} / \mathrm{cm}^{3}$ (amorphous).

(25) Harnau, L.; Costa, D.; Hansen, J. P. Europhys. Lett. 2001, 53, 729.

(26) Li, L.; Harnau, L.; Rosenfeldt, S.; Ballauff, M. Phys. Rev. E 2005, $72,051504$.

(27) Rosenfeldt, S.; et al. ChemPhysChem. 2006, 7, 2097.

(28) Barham, P. J.; Chivers, R. A.; Keller, A.; Martinez-Salazar, J.; Organ, S. J. J. Mater. Sci. 1985, 20, 1625.

(29) Voigt-Martin, I. G.; Mandelkern, L. J. Polym. Sci.: Polym. Phys. $1981,19,1769$.

(30) Sommer, J.-U. Eur. Phys. J. E 2006, 19, 413.

(31) Toda, A. Faraday Discuss. 1993, 95, 129.

(32) Ungar, G.; Putra, E. G. R.; de Silva, D. S. M.; Shcherbina, M. A.; Waddon, A. J. Adv. Polym. Sci. 2005, 180, 45. 